\title{
Ovarian Germline Stem Cells (OGSCs) and the Hippo Signaling Pathway Association with Physiological and Pathological Ovarian Aging in Mice
}

\author{
Jia Lia Fangyue Zhou ${ }^{a}$ Tuochen Zheng ${ }^{b}$ Zezheng Pan ${ }^{a} \quad$ Xia Liang ${ }^{a}$ Jian Huang ${ }^{a}$ \\ Liping Zheng $^{\text {a }}$ Yuehui Zheng ${ }^{\text {a }}$ \\ aMedical Teaching Laboratory Center, Jiangxi Medical College, Nanchang University, Nanchang, bSchool \\ of the 1st Clinical Medical Sciences, Jiangxi Medical College, Nanchang University, Nanchang, China
}

\section{Key Words}

Ovarian germline stem cells • Hippo signaling • Ovarian aging • Mice

\begin{abstract}
Background: The Hippo signaling pathway plays fundamental roles in stem cell maintenance in a variety of tissues and has thus implications for stem cell biology. Key components of this recently discovered pathway have been shown to be associated with primordial follicle activation. However, whether the Hippo signaling pathway plays a role in the development of Ovarian Germline Stem Cells (OGSCs) during physiological and pathological ovarian aging in mice is unknown. Methods: Mice at the age of 7 days (7D), or of 2, 10, or 20 months $(2 \mathrm{M}, 10 \mathrm{M}$, $20 \mathrm{M}$ ) and mice at $2 \mathrm{M}$ treated with TPT and CY/BUS drugs were selected as physiological and pathological ovarian aging models, respectively. Immunohistochemistry was used to assess the development of follicles, and the co-localization of genes characteristic of OGSCs with MST1, LATS2 and YAP1 was assessed by immunofluorescence, western blotting and real-time PCR methods. Results: The Hippo signal pathway and MVH/OCT4 genes were co-expressed in the mouse ovarian cortex. The level and co-localization of LATS2, MST1, MVH, and OCT4 were significantly decreased with increased age, but YAP1 was more prevalent in the mouse ovarian cortex of $2 \mathrm{M}$ mice than 7D mice and was not observed in 20M mice. Furthermore, YAP1, MVH, and OCT4 were gradually decreased after TPT and CY/BUS treatment, and LATS2 mRNA and protein up-regulation persisted in TPT- and CY/BUS-treated mice. However, the expression of MST1 was lower in the TPT and CY/BUS groups compared with the control group. In addition, PYAP1 protein showed the highest expression in the ovarian cortexes of 7D mice compared with $20 \mathrm{M}$ mice, and the value of pYAP1/YAP1 decreased from 7D to 20M. Moreover, pYAP1 decreased in the TPT- and CY/BUS-treated groups, but the value of PYAP1/YAP1 increased in these groups. Conclusion: Taken together, our results show that the Hippo signaling pathway is associated with the changes that take place in OGSCs during physiological and pathological ovarian aging in mice. Thus, the Hippo signaling pathway may be involved in the development schedule of OGSCs.
\end{abstract}

Copyright (C) 2015 S. Karger AG, Basel

L.-P. Zheng

and Y.-H. Zheng

KARGER 125
Room 103, Administration Building, Nanchang University; and Medical Teaching Laboratory Center, Nanchang University, XueFu road 999, Nanchang 330031,Jiangxi Province, (China); E-Mail zhengliping@ncu.edu.cn and E-Mail yuehuizheng@163.com 


\section{Cellular Physiology Cell Physiol Biochem 2015;36:1712-1724

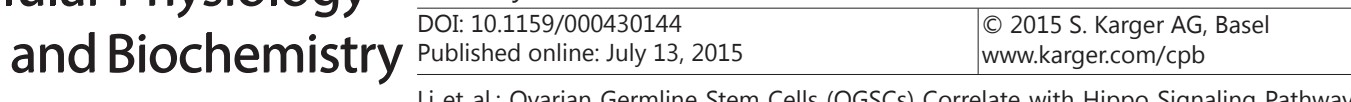 \\ Li et al:: Ovarian Germline Stem Cells (OGSCs) Correlate with Hippo Signaling Pathway in Mice}

\section{Introduction}

Previous studies have shown that primordial follicle depletion is the fundamental reason for physiological and pathological ovarian aging in mammalian females [1]. Historically, it was believed that the majority of the primordial follicle pool remains in a dormant state and declines with age under physiological conditions [2]. At approximately age 50, female fertility begins to decline along with the continued depletion of the primordial follicle pool until the occurrence of menopause [3]. However, certain pathological conditions, such as endometriosis, myomas, and chemotherapy drugs, are thought to cause premature ovarian failure [4-6].

In recent years, this cornerstone understanding of a constant primordial follicle pool was challenged by the work of Johnson et al. [7], who isolated mitotically active germ cells from juvenile and adult mouse ovaries. Based on the rates of oocyte atresia and clearance, these germ cells are needed to continuously replenish the follicle pool. Currently, several research groups have demonstrated the isolation of germline stem cells from the ovarian surface epithelium (OSE) of adult and even menopausal mouse and human ovaries [8-10] and have also successfully isolated and characterized long-term cultured ovarian germline stem cells (OGSCs) from neonatal and adult mouse ovaries [11-17]. However, the OSE layer has also been confirmed to be a normal, naturally present source of oocytes by producing stem cells in the embryonic or fetal period of life, and it is commonly referred to as the "germinal" epithelium [18]. Therefore, some of these recently published studies have shown that OGSCs might be present in the OSE layer.

The Hippo signaling pathway is a recently discovered novel signaling pathway [19]. In mammals, the core of the Hippo pathway comprises two upstream kinases (mammalian Sterile 20-like protein kinase I, MST1 and MST2, for short) and Salvador I (also known as SAV1 or WW45), as well as large tumor suppressor homolog 1 and 2 (LATS1 and LATS2), and YAP1 (Yes-associated protein) [20,21]. Moreover, the Hippo signaling pathway has an essential function in the regulation of cancer stem cell proliferation, differentiation, migration and maturation, as well as the establishment of normal oocyte polarity and egg chamber structure [22-25]. In ovarian CSCs, YAP promotes ovarian CSC tumorigenesis and regulates CSC self-renewal and differentiation [26, 27]. The transcriptional co-activator with PDZbinding motif (TAZ) is a transcriptional effector of the Hippo signaling cascade and regulates cell proliferation and tumorigenesis $[21,28,29]$. In addition, previous studies have shown that YAP acts as an oncogene in a subtype of breast CSCs and lung CSCs, thus serving as a good target for molecular therapies against cancers. The mammalian kinases Mst1 and Mst2 are most abundant in some kinds of tumor tissue systems. MST1 overexpression not only decreases cell proliferation but also induces apoptosis of the human breast cancer cell line MCF-7. Moreover, the overexpression of MST1 can inhibit cell proliferation and promote the cytoplasmic localization and phosphorylation of YAP (Ser127) protein, which may be one of the tumor inhibitory mechanisms of the MST1 gene [30,31]. Consistent with the major roles of MST in cell proliferation and apoptosis, LATS regulates the balance between proliferation and differentiation during adipose development. In addition, LATS knockout leads to the improved efficiency of pluripotent stem cells (IPS), suggesting a barrier to reprogramming and oncogenic transformation [32-34].

The OSE layer involves cell-cell contact and local signaling, which may play very important roles in OGSC growth and development [35]. Previous studies have shown that the Hippo signaling pathway forms a cross network with a variety of factors and multiple signaling pathways such as Notch, TGF $\beta$, c-myc, and PI3K/AKt, which are crucial for the self-renewal and differentiation of stem cells [22, 36-39]. Importantly, several lines of our evidence suggest that the upstream activation of Notch signaling pathways, which have been proven to play a role in germ-line stem cells in ovaries [40, 41], and the Hippo signaling pathway have a spatio-temporal correlation with the size of the primordial follicle pool [42]. Hence, the aim of this study is to reflect the variation in the number of OGSCs and explore 


\section{Cellular Physiology Cell Physiol Biochem 2015;36:1712-1724 \begin{tabular}{l|l} 
Dor: 10.1159/000430144 & $\begin{array}{l}\text { 2015 S. Karger AG, Basel } \\
\text { www.karger.com/cpb }\end{array}$ \\
\hline
\end{tabular} \\ Li et al.: Ovarian Germline Stem Cells (OGSCs) Correlate with Hippo Signaling Pathway in Mice}

the expression changes of the core components of the Hippo pathway (MST1, LATS2, YAP1) during physiological aging and pathological aging of OGSCs in the mice OSE layers.

\section{Materials and Methods}

\section{Animals}

7days, 2 month, 10 months and 20months old KM female mice were housed at the Center of Experimental Animals, Nan Chang University, on a 12-h light: dark cycle and were given access to food (Keao-Xieli, Beijing, China) and water ad libitum. Ovarian failure was induced in one group of 6-weekold KM mice by intraperitoneal injection of cyclophosphamide $(120 \mathrm{mg} / \mathrm{kg})$ and busulfan $(12 \mathrm{mg} / \mathrm{kg})$ simultaneously. However, ovarian failure was induced by the other group by infusing the stomachs of the mice with Tripterygium wilfordii polycoride tablets. According to the previous reported, we used lavage needle, gently gavaged needle from the mouth of mice, arrived the esophagus and pushed TPT liquid (0.6 $\mathrm{mg} /(100 \mathrm{~g} / \mathrm{d})$ for $9 \mathrm{~d})$. The controls were intraperitoneally injected with saline or had it infused into the stomach. All procedures (including the use of animals in this study) were reviewed and approved by the ethical committee of Nan Chang University.

\section{Histological analysis of ovarian tissue}

For the evaluation of follicles, ovaries were fixed in $4 \%$ paraformaldehyde and prepared as paraffin blocks; 5-mm sections were obtained serially and stained with hematoxylin-eosin (HE). Immunostaining of ovaries from 7 days, 2 month and 20 months old mice were performed according to previously published procedures [8]. The primary antibodies used in this study were as follows: anti-MVH (1:100, ab27591), anti-OCT4 (1:100, ab18976), anti-YAP1 (1:100, ab56701), anti-LATS2 (1:100, ab54073), and anti-MST1 (1:100, ab51134). The secondary antibodies used in this study were goat anti-mouse and goat anti-rabbit IgG conjugated with fluorescein isothiocyanate at dilution of 1:200 (proteintech, China). Then, the tissues were stained with DAPI, and all of the images were taken using a NIKON Eclipse 80i microscope.

\section{Quantitative real-time PCR}

The total RNA of different stages of mice and treated ovarian surface epithelium was extracted with TRIzol reagent (TaKaRa). cDNA was synthesized using a PrimeScript RT reagent Kit with gDNA Eraser (TaKaRa). Quantitative RT-PCR was performed using an ABI7000 PCR instrument (Applied Biosystems, Foster City, CA, USA) using two-stage program parameters provided by the manufacturer. The primers used for PCR are listed in Table 1. Every sample was amplified in triplicate to normalize system and pipetting errors, and GAPDH was used as the reference gene to normalize the expression results.

Table 1. Primers used for quantitative-PCR

\begin{tabular}{clcc}
\hline Gene & \multicolumn{1}{c}{ Primer Sequence (5'-3') } & Sequence number & Amplified fragment length (bp) \\
\hline MVH & F:GTGTATTATTGTAGCACCAACTCG & NM_010029.2 & 147 \\
& R:CACCCTTGTACTATCTCTCGAACT & & 198 \\
OCT4 & F: AGCTGCTGAAGCAGAAGAGG & NM_013633 & \\
& R: GGTTCTCATTGTTGTCGGCT & & 117 \\
MST1 & F:CAGCCCGAGGAAGTGTTTGA & XM_006499963.1 & \\
& R:CACGGGCACTTGCTTGATTG & & 146 \\
LATS2 & F:CTTTGCTTCCCTCGGACAGT & NM_010690.1 & \\
& R:AACTGGGTGTAGTCAGGGGT & & \\
YAP1 & F:TCCAACCAGCAGCAGCAAAT & NM_001171147 & 102 \\
& R:TTCCGTATTGCCTGCCTGCCGAAA & & \\
GAPDH & F:ACGTGCCGCCTGGAGAAAC & XM_001476707.3 & \\
& R:GTCCTCAGTGTAGCCCAAGATGC & & \\
\hline & & & \\
\end{tabular}




\section{Cellular Physiology Cell Physiol Biochem 2015;36:1712-1724 \begin{tabular}{l|l} 
and Biochemistry Published onIIne: July 13, 2015 & $\begin{array}{l}\text { C) 2015 S. Karger AG, Basel } \\
\text { www.karger.com/cpb }\end{array}$ \\
\hline
\end{tabular} \\ Li et al.: Ovarian Germline Stem Cells (OGSCs) Correlate with Hippo Signaling Pathway in Mice}

Western blot analysis

Total protein was extracted from different ovarian tissue with RIPA lysis solution (Beyotime, P0013C). The proteins were subjected to SDS-PAGE (sodium dodecyl sulfate-polyacrylamide gel electrophoresis) and then transferred to PVDF membranes (Millipore Corp., Bedford, MA) and processed per the antibody manufacturer's instructions. The antibodies used for western blotting were anti-YAP1 (1:100, ab56701, Abcam), anti-S127 phosphorylated-YAP1 (1:10000, ab76252, Abcam), anti-MST1 (1:10000, ab51134, Abcam), anti-LATS2 (1:100, ab54073, Abcam), anti-MVH (1:5000, ab27591, Abcam), anti-OCT4 (1:100, ab18976, Abcam) and anti-GAPDH (1:1000, ab1801, Abcam). The results were analyzed by gel imaging and analysis system and converted to a semi-quantitative data by Gelscan software. The level of GAPDH was quantified at the same time as an internal quantitative control. Each experiment was repeated at least three times.

\section{Statistical methods}

All analyses were performed using GraphPad Prism 5.0 (GraphPad Software, Inc., San Diego, CA). The statistical comparisons among different groups were analyzed by Student's paired t-test. The threshold of $\mathrm{p}$ $<0.05$ was considered significant; $\mathrm{p}<0.01$ and $\mathrm{p}<0.001$ were each considered extremely significant.

\section{Results}

Expression of OGSC specific genes during physiological ovarian aging in mice

To confirm follicle depletion in the 7D, 2M and 20M mice, we performed histological analysis of the ovaries. As seen in Fig. 1A, as the age of the mice increased, the number of primordial and mature follicles was reduced, while the number of atretic follicles increased gradually $(\mathrm{p}<0.05, \mathrm{p}<0.01$, and $\mathrm{p}<0.001)$. Meanwhile, the corpus luteum level was highest in $2 \mathrm{M}$ mice $(\mathrm{p}<0.001)$. The mature follicles had most the significant difference in $2 \mathrm{M}$ and $20 \mathrm{M}$ mice $(\mathrm{p}<0.001)$.

Western blotting and RT-PCR assays were used to detect MVH/OCT4 expression in the ovarian cortexes of 7D, 2M and 20M mice. The western blot results showed that the protein expression of MVH/OCT4 was decreased significantly as mice aged ( $p<0.001$ ) (Fig. 1B), and the relative mRNA expression of MVH/OCT4 showed a significant decrease among $2 \mathrm{M}, 10 \mathrm{M}$ and $20 \mathrm{M}$ mice compared with the 7D mice $(\mathrm{p}<0.01, \mathrm{p}<0.001)$. MVH expression decreased significantly $(\mathrm{p}<0.05, \mathrm{p}<0.01$ ) among the $10 \mathrm{M}$ and $20 \mathrm{M}$ mice compared with the $2 \mathrm{M}$ mice, but OCT4 expression was significantly reduced when comparing the $2 \mathrm{M}$ and $20 \mathrm{M}$ mice $(\mathrm{p}$ $<0.05$ ) (Fig. 1C). Subsequently, we obtained micrographs of mouse ovarian cortexes in 7D, $2 \mathrm{M}$ and 20M mice to determine the MVH/OCT4 gene expression. As shown in Fig.1D, MVH/ OCT4 expression was highest in ovarian cortexes from 7D mice, which then significantly down-regulated from $2 \mathrm{M}$ to $20 \mathrm{M}$, with almost no expression at 20M. These data suggest that ovarian stem cells are present in the ovarian cortex at different stages and that their levels gradually decrease with age in mice.

\section{Expression of OGSC specific genes during pathological ovarian aging in mice}

In the TPT and CY/BUS mouse models, we also showed that the numbers of primordial follicles, mature follicles and corpus luteum were decreased significantly $(p<0.001)$. The number of atretic follicles increased gradually compared with the control group $(p<0.001)$, but there was no significant difference between the control and CY/BUS-treated mice ( $p<$ 0.05) (Fig. 2A). However, we found that the numbers of primordial follicles, mature follicles, atretic follicles and corpus luteum in the CY/BUS group were significantly decreased compared with those of the TPT group ( $p<0.05, p<0.01, p<0.001)$. HE-stained sections of ovaries revealed fewer or almost no viable follicles in chemotherapy-treated ovaries.

We established western blot and RT-PCR methods to assess whether MVH/OCT4 expression varies in TPT- and CY/BUS-treated mouse ovarian cortexes. Western blot analysis of MVH/OCT4 revealed that the expression levels were significantly lower compared with the control group $(\mathrm{p}<0.01, \mathrm{p}<0.001)$ but were not significantly different between the 
A
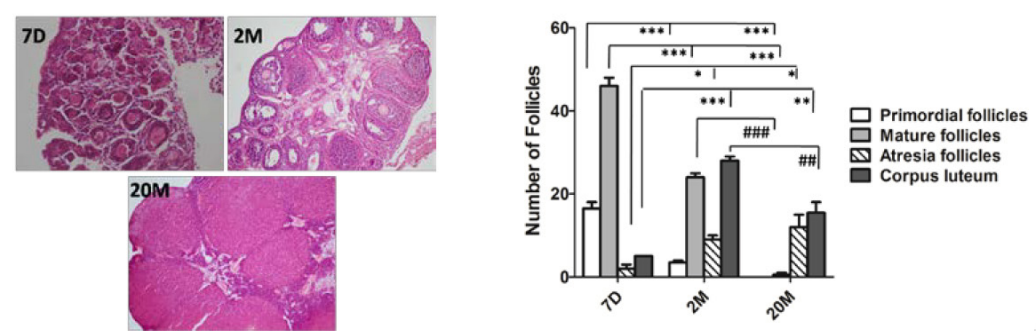

B
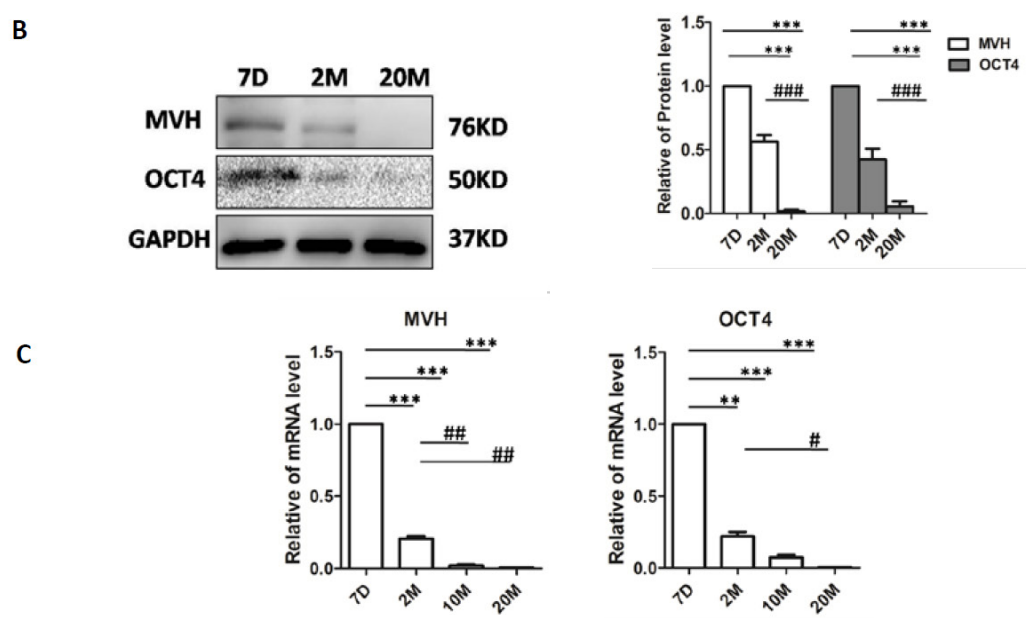

D

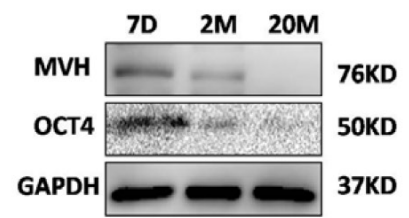

$20 \mathrm{M}$
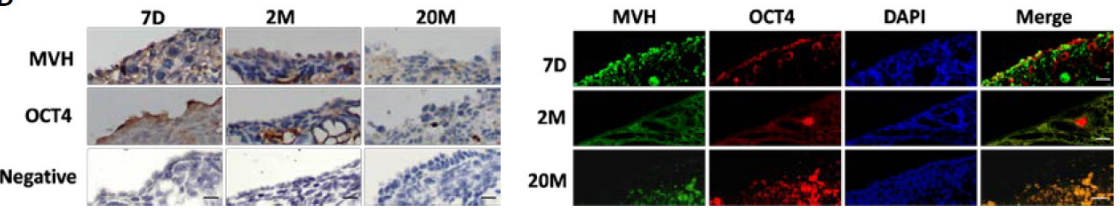

Fig. 1. The dynamic changes of germ-line stem cells during physiological ovarian aging in the mouse ovarian cortex. A: The detection of ovarian follicle depletion by HE-staining. The number of primordial and mature follicles is reduced with increased age, but atretic follicles increased gradually; B: Western blot analysis showed that the expression of MVH/OCT4 protein decreased as mouse age increased. C: Quantitative RT-PCR analyses showed the level of MVH/OCT4 mRNA decreased as mouse age increased. The results are presented as the mean \pm SD. ${ }^{*} \mathrm{p}<0.05,{ }^{* *} \mathrm{p}<0.01$, ${ }^{* * *} \mathrm{p}<0.001$, compared with the 7D group. \# $\mathrm{p}<0.05$, \#\# $\mathrm{p}<0.01$, \#\#\# p $<0.001$, compared with the 2M group. D: Micrographs depict MVH/OCT4 as detected by anti-MVH antibody (red) and anti-OCT4 antibody (green). The nuclei were stained by DAPI (blue). Scale bars represent $20 \mu \mathrm{m}$.

ovarian cortexes of TPT- and CY/BUS-treated mice (p>0.05) (Fig. 2B). The relative mRNA expression of MVH/OCT4 showed a significant decrease compared with the control group ( $p$ $<0.05$, p < 0.01) (Fig. 2C). Meanwhile, the MVH levels were significantly different in the TPT and CY/BUS groups relative to the control $(\mathrm{p}<0.05)$. In addition, double immunofluorescent staining showed the expression of MVH/OCT4 proteins in the ovarian cortexes of control and TPT- and CY/BUS-treated mice. A lower level of MVH was associated with a significant down-regulation in the pathological aging of the ovarian cortex of mice compared with the control. Similar to MVH, the expression of OCT4 was also lower in TPT- and CY/BUS-treated mice than in control mice (Fig. 2D). These data also suggest that OSEs are present in the mouse ovarian cortex during pathological ovarian aging and their levels are decreased in TPT- and CY/BUS-treated mice.

\section{KARGER}


A

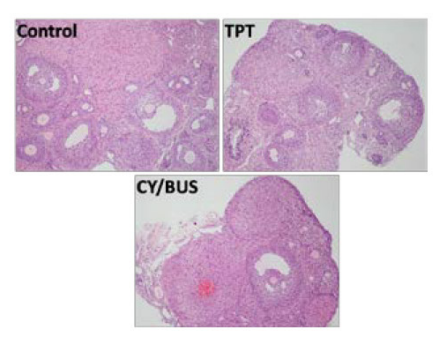

B

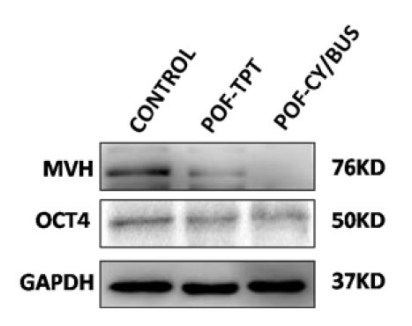

c

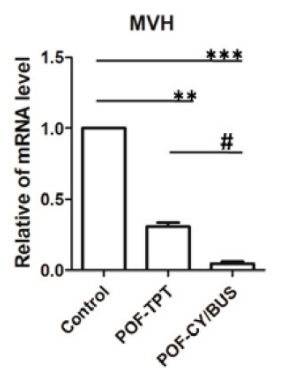

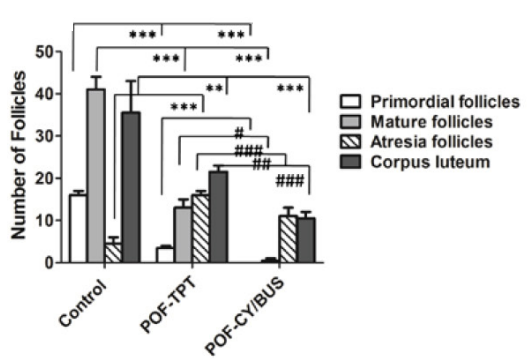
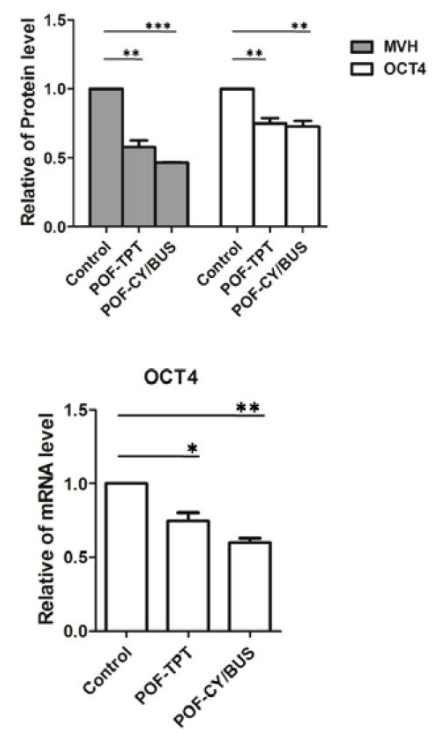

D
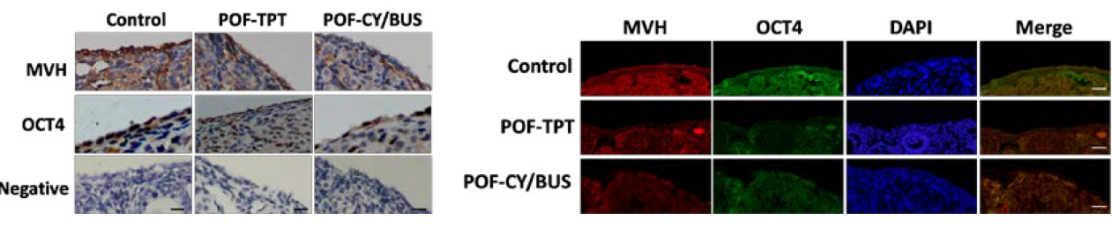

Fig. 2. The associations of the level of germ-line stem cells during pathological ovarian aging in the mouse ovarian cortex. A: The detection of ovarian follicle depletion by HE-staining. The number of primordial and mature follicles was reduced in TPT- and CY/BUS-treated mice, but atretic follicles increased gradually; B: Western blot analysis showed the expression of MVH/OCT4 protein in TPT- and CY/BUS-treated mice compared to the control. C: Quantitative RT-PCR analyses showed the level of MVH/OCT4 mRNA in TPT and $\mathrm{CY} /$ BUS-treated mice compared to the control. The results are presented as the mean $\pm \mathrm{SD}$. ${ }^{*} \mathrm{p}<0.05$, ${ }^{* *} \mathrm{p}<$ $0.01,{ }^{* * *} \mathrm{p}<0.001$, compared with the control group. $\# \mathrm{p}<0.05$, \#\# p $<0.01$, \#\#\# p $<0.001$, compared with the TPT group. D: Micrographs depict MVH/OCT4 as detected by anti-MVH antibody (red) and anti-OCT4 antibody (green). The nuclei were stained by DAPI (blue). Scale bars represent $20 \mu \mathrm{m}$.

\section{Expression of Hippo signaling components in ovarian cortex during physiological ovarian aging}

To identify the expression of Hippo signaling in the ovarian cortex, we extracted the total RNA and protein from ovaries of 7D, 2M, 10M and 20M mice. The figures showed that the mRNA and protein expression of LATS2 and MST1 were significantly decreased with increased age $(\mathrm{p}<0.05, \mathrm{p}<0.01, \mathrm{p}<0.001)$ (Fig. 3A, C). However, the relative mRNA expression of YAP1 gradually increased in the ovaries from 7D to $10 \mathrm{M}$ mice, whereas the YAP1 level in 20M mice was significantly decreased compared with the level in $10 \mathrm{M}$ mice ( $\mathrm{p}$ $<0.05$ ) (Fig. 3A). The concentration of YAP1 protein was also remarkably different between 


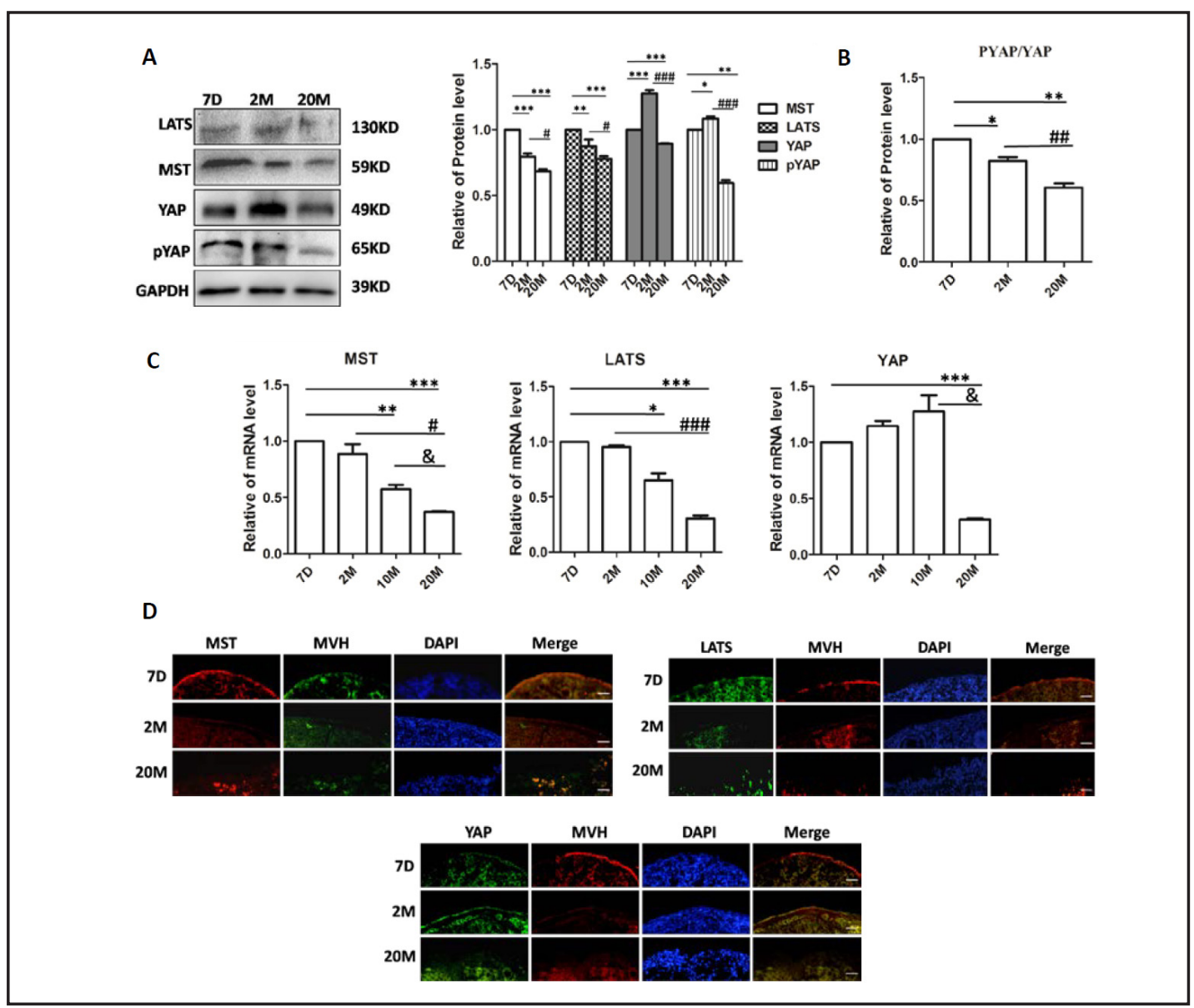

Fig. 3. The relationship between the levels of Hippo signaling genes in the germ-line stem cells of the ovarian cortex and physiological ovarian aging in mice. A: Analysis of MST1, LATS2 and YAP1 expression in the ovarian cortexes of 7D, 2M and 20M mice by western blot methods. B: Analysis of MST1, LATS2 and YAP1 expression in the ovarian cortexes of 7D, 2M, 10M and 20M mice by RT-PCR assays. C: The value of pYAP1/ YAP1. The results are presented as the mean $\pm \mathrm{SD}$. $* \mathrm{p}<0.05$, ** $\mathrm{p}<0.01$, *** $\mathrm{p}<0.001$, compared with the 7D group. \# $\mathrm{p}<0.05$, \#\# $\mathrm{p}<0.01$, \#\#\# $\mathrm{p}<0.001$, compared with the $2 \mathrm{M}$ group. \& $\mathrm{p}<0.05, \& \& \mathrm{p}<0.01$, $\& \& \& \mathrm{p}<0.001$, compared with the 10M group. D: Micrographs depict MST1, LATS2, and YAP1 in the mouse ovaries of 7D, 2M and 20M mice as detected by anti-MST1 antibody (red), anti-LATS2 antibody (green), and anti-YAP1 antibody (green). The nuclei were stained by DAPI (blue). Scale bars represent $20 \mu \mathrm{m}$.

7D, 2M and 20M mice ( $\mathrm{p}<0.001$ ) (Fig. 3A). In addition, the protein expression of pYAP1 was the highest in the ovarian cortex of 7D mice and lowest in 20M mice (Fig. $3 \mathrm{~A})(\mathrm{p}<0.05, \mathrm{p}$ $<0.01$ ). As the age of the mice increased, the value of pYAP1/YAP1 decreased in $20 \mathrm{M}$ mice compared to 7D mice (Fig. 3B) $(p<0.05, p<0.01)$. These results indicate that the effectors of the Hippo pathway and pYAP1 are correlated with the proliferative ability of OGSCs.

As shown in Fig. 3D, MST1, LATS2 and YAP1 were distinctly localized in the mouse ovarian cortexes. The magnitude of incorporated LATS2 or MST1 in the ovarian cortexes of $2 \mathrm{M}$ and $20 \mathrm{M}$ mice was visibly lower than that observed in 7D mice. Conversely, YAP1 accumulation was greater in the ovarian cortexes of 2M mice compared to 7D mice, and YAP1 was not observed in $20 \mathrm{M}$ mice.

Expression of Hippo signaling components in ovarian cortex during pathological ovarian aging

Given the above results, we next assessed the expression of Hippo signaling pathway members during pathological aging in the mouse ovarian cortex caused by TPT and CY/ 
A

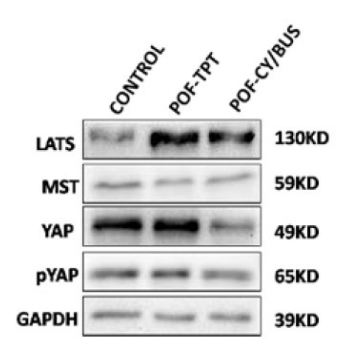

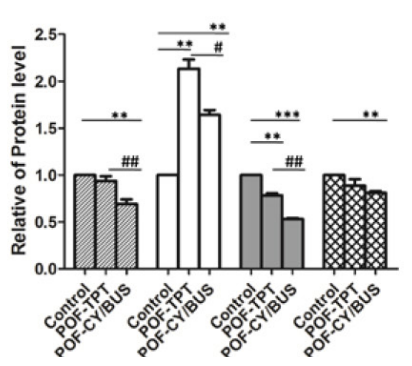

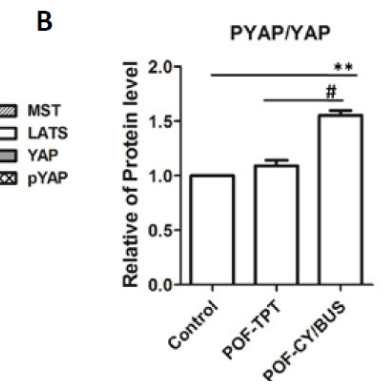

c
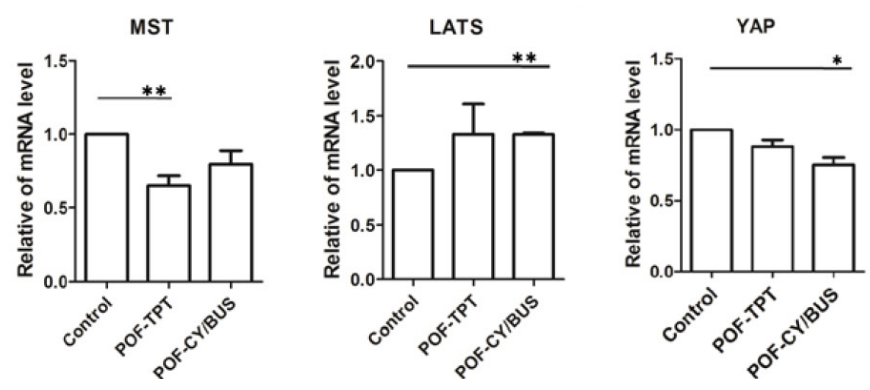

D
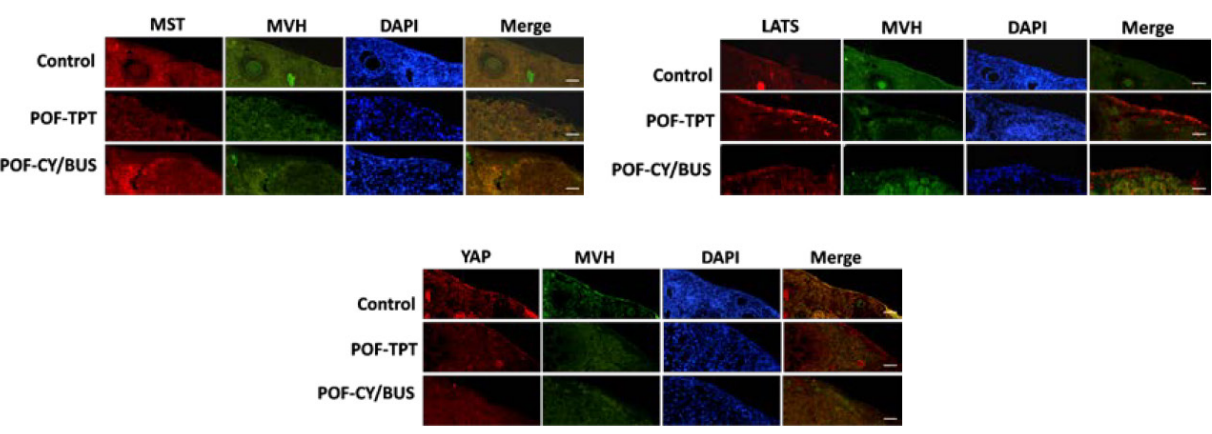

Fig. 4. The relationship between the levels of Hippo signals genes in the germ-line stem cells of the ovarian cortex and pathological ovarian aging in mice. A: Analysis of MST1, LATS2 and YAP1 expression in the ovarian cortexes of control, TPT and CY/BUS groups by western blot methods. B: Analysis of MST1, LATS2 and YAP1 expression in the ovarian cortexes of control, TPT and CY/BUS mice by RT-PCR assays. C: The value of pYAP1/YAP1. The results are presented as the mean \pm SD. ${ }^{*} \mathrm{p}<0.05,{ }^{* *} \mathrm{p}<0.01,{ }^{* * *} \mathrm{p}<0.001$, compared with the 7D group. \# $\mathrm{p}<0.05$, \#\# $\mathrm{p}<0.01$, \#\#\# $\mathrm{p}<0.001$, compared with the control group. \& $\mathrm{p}<0.05, \& \& \mathrm{p}<0.01, \& \& \& \mathrm{p}<0.001$, compared with the TPT group. D: Micrographs depict MST1, LATS2, and YAP1 during in the ovaries of control, TPT and CY/BUS mice as detected by anti-MST1 antibody (red), anti-LATS2 antibody (green), and anti-YAP1 antibody (green). The nuclei were stained by DAPI (blue). Scale bars represent $20 \mu \mathrm{m}$.

BUS. As shown in Fig.4, the mRNA and protein expression levels of YAP1 were significantly decreased between the control and CY/BUS-treated groups ( $p<0.05, p<0.01, p<0.001)$, whereas the expression of YAP1 did not differ significantly between the control and TPTtreated groups. LATS2 mRNA and protein up-regulation persisted when mice were treated with TPT or CY/BUS ( $p<0.05, p<0.01$ ). However, MST1 expression was different from the level of LATS2 expression in that the relative mRNA expression of MST1 was lower in the TPT- or CY/BUS-treated mice compared with the control group $(\mathrm{p}<0.05)$. In addition, the total protein level of MST1 was decreased $(\mathrm{p}<0.01)$, and there was no difference in the 


\section{Cellular Physiology Cell Physiol Biochem 2015;36:1712-1724

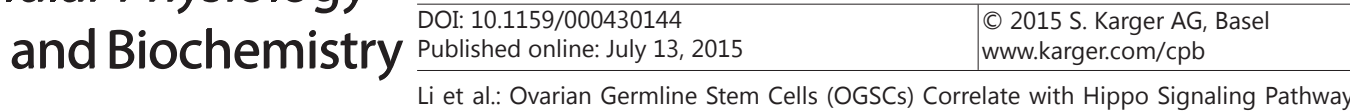 in Mice}

mRNA or protein expression in the CY/BUS or TPT groups compared with the control group ( $>0.05$ ) (Fig. 4A, 4C). Similar to YAP1, pYAP1 protein also decreased in the TPT- or CY/BUStreated group (Fig. 4B) ( $<<0.01)$, and pYAP1 did not differ significantly between the control and TPT-treated group. In addition, the value of pYAP1/YAP1 increased between the control and CY/BUS-treated group $(\mathrm{p}<0.05, \mathrm{p}<0.01)$ and was significantly different between the TPT and CY/BUS groups $(\mathrm{p}<0.05)$.

Subsequently, the micrographs showed that MVH was also expressed in the mouse ovarian cortex and was down-regulated in the TPT and CY/BUS groups compared with control group. Like the IF results in the mouse ovarian cortex during physiological ovarian aging, the Hippo genes were also present in the mouse ovarian cortex during pathological ovarian aging. Specifically, the most intense staining was that of LATS2 in TPT-treated mice than CY/BUS and control groups. Moreover, the intensity of YAP1 and MST1 staining in TPTor CY/BUS-treated mice was distinctly lower than control (Fig. 4D).

\section{Discussion}

Morphometric analysis of the ovaries showed that the number of primordial follicles make up most of the total follicles in 7D mice ovaries. With further growth and differentiation, primordial follicles are gradually replaced by different levels of follicles (Fig. 1A). Meanwhile, there were no normal follicles except atretic follicles in TPT- and CY/BUStreated ovaries, unlike the control (Fig. 2A). Therefore, we chose these physiological and pathological ovarian aging models as experimental groups to reflect the variation in Hippo signaling pathway components during the development of OGSCs over the reproductive life of female mice.

To identify and confirm the presence of OGSCs in different stages of mouse ovaries, we located ovarian cells positive for mouse vasa homologue (MVH) and OCT4 protein, which are expressed exclusively in germ cells and pluripotent stem cells, respectively $[43,44]$. The immunocytochemical results confirmed that MVH and OCT4 were concurrently co-expressed in different stages of mouse ovarian cortex (Fig. 1D). Moreover, we have provided evidence for the expression of MVH/OCT4 genes and proteins in the different stages of mouse ovaries (Fig1 A, B, C). MVH and OCT4 were maximally expressed in 7D mice ovaries compared with the other groups, but were also expressed in $2 \mathrm{M}$ mice, and minimally expressed at $10 \mathrm{M}$. Up to 20M, there was almost no detectable MVH or OCT4 expression. Briefly, OGSCs were observed in the OSE layers, and the number of OGSCs gradually decreased from 7D to 20M. However, previous studies have shown that OGSCs can complement the consumption of primordial follicles via proliferation and differentiation [45]. However as aging progresses the number of OGSCs decreases, which may cause the depletion rate of the follicle pool to exceed the supplementation rate during the fertility exuberant female mice,. These changes may be the root cause of menopause symptoms around age 50 .

Our recent results revealed that the Hippo signaling pathway is expressed in mouse ovaries and the expression of the core components of the Hippo pathway change during mouse follicular development [42]. In this study, as seen in Fig. 3, MST1 and LATS2 were most highly expressed in the ovarian cortexes of 7D mice, but YAP1 was more abundant in $2 \mathrm{M}$ than in 7D mice and was absent in 20M mice. Whereas the primordial follicles start developing unceasingly with age, follicular growth is accompanied by oocyte maturation and granular cell proliferation. MST1 and LATS2 were down-regulated during follicle formation and depletion with age, but the expression level of YAP1 was up-regulated at all time points until 10M. Based on this result, we hypothesized that the 7D mice were not in the gonadotropin phase because they could not yet regulate the levels of stem cells and OGSC proliferation remained in the stationary phase of the cell cycle [46]. However, there is increased differentiation of follicles with advanced age when gonadotropin levels are elevated and the proliferation rate of OGSCs is accelerated. This state is maintained until the age of $10 \mathrm{M}$. Therefore, it is believed that the phenomenon may be involved in the expression 


\section{Cellular Physiology Cell Physiol Biochem 2015;36:1712-1724

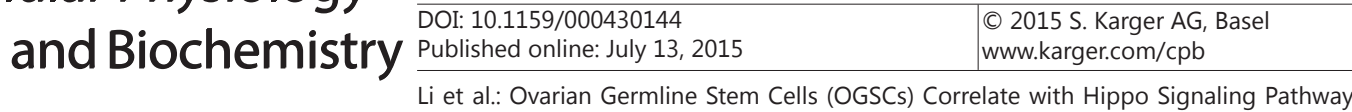 in Mice}

change of YAP1. As shown in Fig. 3D, MST1, LATS2, YAP1 and MVH were specifically coexpressed in the mouse ovarian cortex, and the levels of MST1 and LATS2 also decrease among 7D, 2M and 20M mice, unlike the expression of YAP1. Moreover, the change in pYAP1 expression is similar to that of YAP1, but the ratio of pYAP1/YAP1 decreased significantly among 7D, 2M and 20M mice. High cell-density activates the Hippo pathway kinases and promotes the phosphorylation of YAP1 on serine 127, which leads to its nuclear export and growth inhibition $[47,48]$. Furthermore, the results show that Hippo signaling genes are active in different stages of mouse ovarian cortex, and OGSCs had a positive correlation with the level of MST1 and LATS2 and were contrary to the changes in YAP1 expression.

Likewise, MVH and OCT4 were co-expressed and these levels were remarkably decreased in the ovarian cortex of TPT- and CY/BUS-treated mice (Fig2). The expression patterns presented here are similar to that shown in a previous study, which showed that ovarian stem cells are present in the OSE during ovarian failure and patients with no naturally present follicles and oocytes $[8,10]$. Previous studies have suggested that differences in follicle depletion are caused by two drugs: TPT and CY/BUS $[49,50]$. However, there is little information on the expression of MVH and OCT4 in the ovaries of TPT- and CY/BUS-treated mouse models. In our study, we demonstrated that MVH and OCT4 are present in the TPTand CY/BUS-treated mouse ovarian cortexes, and the changes of OGSCs in TPT- and CY/BUStreated mice are consistent with the results seen for physiological ovarian aging in mice. Figs. $2 \mathrm{~B}, \mathrm{C}$ suggest that there is almost no difference in the effect of these two on the presence and quantity of OGSCs. In addition, the expression of OCT4 in the mouse ovarian cortex of both the TPT- and CY/BUS-treated mice was higher than that in 20M mice, suggesting that there are some putative stem cells in the ovarian cortex of mice undergoing pathological ovarian aging. Given these results, it has been speculated that OGSC proliferation suppression may be the key cause of follicular depletion by TPT and CY/BUS treatment and may also be the core cause of pathological ovarian aging caused by chemical treatment in cancer patients.

We assessed the chemotherapy-induced ovarian follicular loss in mice, arguing that Hippo signaling factors are important for the development of OGSCs. However, the mechanisms involved in the changes of Hippo signaling genes during chemotherapy-induced ovarian follicular loss in mice remain obscure. MST1, LATS2 and YAP1 were significantly detected in the ovarian cortex of the TPT- and CY/BUS-treated mice and the control group, respectively. The results of Fig. 4A, B indicate that the expression of MST1 and YAP1 were decreased after TPT and CY/BUS treatment, whereas the mRNA and protein levels of LATS2 were significantly higher in the TPT- and CY/BUS-treated group compared to the control. The amount of MST1, LATS2 and YAP1 protein expression was significantly different within the TPT- and CY/BUS-treated mice ovaries, but the amount of mRNA expression for MST1, LATS2 and YAP1 are not significantly different from the control levels. However, we can hypothesize about the role of Hippo signaling in pathological ovarian aging as follows: First, RNA is converted into protein by a complex regulatory process $[51,52]$. These genes in the TPT- and CY/BUS-treated mouse ovaries are disturbed by these drugs, leading to a suspension of translation and resulting in a significant decrease in the protein levels of Hippo signaling components. Second, post-translational processing takes place [53]. If protein processing after translation is not correct, the protein may be degraded by endopeptidase, and activity may also be changed or lead to the loss of required core components for translation into protein in the TPT- and CY/BUS-treated mouse ovaries. This problem will finally cause the change in protein levels. It is known that MST and LATS are suppressor genes in the Hippo signaling pathway $[25,28,54]$. Interestingly, MST1 mRNA and protein levels are not consistent with the expression of LATS in the TPT- and CY/BUS -treated mouse ovaries. Based on all of these previous studies, it seems that different protein kinases are involved in activating MST and LATS [55], resulting in the observation of an inverse trend in MST1 protein expression compared to the level of LATS2. In addition, the proteins of pYAP1 were decreased in the TPT- and CY/BUS -treated group, as shown using western blotting, and the ratio of pYAP1/YAP 1increased significantly. 


\section{Cellular Physiology Cell Physiol Biochem 2015;36:1712-1724 \begin{tabular}{l|l} 
Dor: 10.1159/000430144 & $\begin{array}{l}\text { 2015 S. Karger AG, Basel } \\
\text { www.karger.com/cpb }\end{array}$ \\
\hline
\end{tabular} \\ Li et al.: Ovarian Germline Stem Cells (OGSCs) Correlate with Hippo Signaling Pathway in Mice}

In conclusion, this study demonstrated that Hippo signaling genes are expressed in OGSCs, and the number of OGSCs correlates with the dynamic changes of Hippo signaling. These findings confirmed that the Hippo signaling pathway may be involved in dictating the development schedule of OGSCs. In a follow-up study, we plan to use OGSC line as a useful tool to study Hippo signaling molecules and the effect on OGSCs transfected by lentivirus to mice ovaries. These future studies could be used for further research aimed at the autologous treatment of ovarian infertility and degenerative diseases.

\section{Acknowledgments}

This work was supported by the National Nature Science Foundation (No.81260098, No.81360100) and the Excellence 555 Engineering of Jiangxi province. We are thankful to the Life Sciences Institute of Nanchang University for providing a high standard of scientific research conditions. We also thank all of the members in our lab.

\section{Disclosure Statement}

None.

\section{References}

1 Macklon NS, Fauser BC: Aspects of ovarian follicle development throughout life. Horm Res 1999;52:161170.

2 McGee EA, Hsueh AJ: Initial and cyclic recruitment of ovarian follicles. Endocr Rev 2000;21:200-214.

3 Adhikari D, Liu K: Molecular mechanisms underlying the activation of mammalian primordial follicles. Endocr Rev 2009;30:438-464.

4 Meirow D, Biederman H, Anderson RA, Wallace WH: Toxicity of chemotherapy and radiation on female reproduction. Clin Obstet Gynecol. 2010;53:727-739.

5 Wu X, Cai H, Kallianpur A, Li H, Yang G, Gao J, Xiang YB, Ji BT, Yu T, Zheng W, Shu XO: Impact of premature ovarian failure on mortality and morbidity among chinese women. PloS one 2014;9:e89597.

-6 Broekmans FJ, Soules MR, Fauser BC: Ovarian aging: Mechanisms and clinical consequences. Endocr Rev 2009;30:465-493.

7 Johnson J, Canning J, Kaneko T, Pru JK, Tilly JL: Germline stem cells and follicular renewal in the postnatal mammalian ovary. Nature 2004;428:145-150.

-8 Virant-Klun I, Skutella T, Stimpfel M, Sinkovec J: Ovarian surface epithelium in patients with severe ovarian infertility: A potential source of cells expressing markers of pluripotent/multipotent stem cells. J Biomed Biotechnol 2011;2011:381928.

-9 Weissman A, Gotlieb L, Colgan T, Jurisicova A, Greenblatt EM, Casper RF: Preliminary experience with subcutaneous human ovarian cortex transplantation in the NOD-SCID mouse. Biol Reprod 1999;60:14621467.

10 Virant-Klun I, Zech N, Rozman P, Vogler A, Cvjeticanin B, Klemenc P, Malicev E, Meden-Vrtovec H: Putative stem cells with an embryonic character isolated from the ovarian surface epithelium of women with no naturally present follicles and oocytes. Differentiation 2008;76:843-856.

11 Pacchiarotti J, Maki C, Ramos T, Marh J, Howerton K, Wong J, Pham J, Anorve S, Chow YC, Izadyar F: Differentiation potential of germ line stem cells derived from the postnatal mouse ovary. Differentiation 2010;79:159-170.

$>12$ Shi L, Wu J: Epigenetic regulation in mammalian preimplantation embryo development. Reprod Biol Endocrinol 2009;7:59.

13 Zou K, Yuan Z, Yang Z, Luo H, Sun K, Zhou L, Xiang J, Shi L, Yu Q, Zhang Y, Hou R, Wu J: Production of offspring from a germline stem cell line derived from neonatal ovaries. Nat Cell Biol 2009;11:631-636. 


\section{Cellular Physiology Cell Physiol Biochem 2015;36:1712-1724 \begin{tabular}{l|l} 
Dor: 10.1159/000430144 & $\begin{array}{l}\text { 2015 S. Karger AG, Basel } \\
\text { www.karger.com/cpb }\end{array}$ \\
\hline
\end{tabular} \\ Li et al.: Ovarian Germline Stem Cells (OGSCs) Correlate with Hippo Signaling Pathway in Mice}

14 Imudia AN, Wang N, Tanaka Y, White YA, Woods DC, Tilly JL: Comparative gene expression profiling of adult mouse ovary-derived oogonial stem cells supports a distinct cellular identity. Fertil Steril 2013;100:14511458.

15 Woods DC, Tilly JL: Isolation, characterization and propagation of mitotically active germ cells from adult mouse and human ovaries. Nat Protoc 2013;8:966-988.

16 White YA, Woods DC, Takai Y, Ishihara O, Seki H, Tilly JL: Oocyte formation by mitotically active germ cells purified from ovaries of reproductive-age women. Nat med 2012;18:413-421.

$\$ 17$ Woods DC, White YA, Tilly JL: Purification of oogonial stem cells from adult mouse and human ovaries: An assessment of the literature and a view toward the future. Reprod Sci 2013;20:7-15.

$\checkmark 18$ Evans HM, Swezy 0: Ovogenesis and the normal follicular cycle in adult mammalia. Cal West Med 1932;36:60.

19 Zhang ZW, Men T, Feng RC, Li YC, Zhou D, Teng CB: miR-375 inhibits proliferation of mouse pancreatic progenitor cells by targeting YAP1. Cell Physiol Biochem 2013;32:1808-1817.

20 Zhao B, Lei QY, Guan KL: The hippo-yap pathway: New connections between regulation of organ size and cancer. Curr Opin Cell Biol 2008;20:638-646.

21 Lei QY, Zhang H, Zhao B, Zha ZY, Bai F, Pei XH, Zhao S, Xiong Y, Guan KL: Taz promotes cell proliferation and epithelial-mesenchymal transition and is inhibited by the hippo pathway. Mol Cell Biol 2008;28:24262436.

-22 Polesello C, Tapon N: Salvador-warts-hippo signaling promotes drosophila posterior follicle cell maturation downstream of notch. Curr Biol 2007;17:1864-1870.

-23 Barry ER, Camargo FD: The hippo superhighway: Signaling crossroads converging on the hippo/yap pathway in stem cells and development. Curr Opin Cell Biol 2013;25:247-253.

-24 Karpowicz P, Perez J, Perrimon N: The hippo tumor suppressor pathway regulates intestinal stem cell regeneration. Development 2010;137:4135-4145.

25 Hao J, Zhang Y, Jing D, Li Y, Li J, Zhao Z: Role of hippo signaling in cancer stem cells. J Cell Physiol 2014;229:266-270.

26 Hall CA, Wang R, Miao J, Oliva E, Shen X, Wheeler T, Hilsenbeck SG, Orsulic S, Goode S: Hippo pathway effector yap is an ovarian cancer oncogene. Cancer Res 2010;70:8517-8525.

27 Zhang X, George J, Deb S, Degoutin JL, Takano EA, Fox SB, group AS, Bowtell DD, Harvey KF: The hippo pathway transcriptional co-activator, yap, is an ovarian cancer oncogene. Oncogene 2011;30:2810-2822.

28 Pan D: The hippo signaling pathway in development and cancer. Dev Cell 2010;19:491-505.

29 Jeong GO, Shin SH, Seo EJ, Kwon YW, Heo SC, Kim KH, Yoon MS, Suh DS, Kim JH: TAZ mediates lysophosphatidic acid-induced migration and proliferation of epithelial ovarian cancer cells. Cell Physiol Biochem 2013;32: 253-263.

-30 Xu C, Liu C, Huang W, Tu S, Wan F: Effect of mst1 overexpression on the growth of human hepatocellular carcinoma hepg2 cells and the sensitivity to cisplatin in vitro. Acta Biochim Biophys Sin 2013;45:268-279..

31 Li P, Chen Y, Mak KK, Wong CK, Wang CC, Yuan P: Functional role of mst1/mst2 in embryonic stem cell differentiation. PloS one 2013;8:e79867.

-32 Zhang J, Smolen GA, Haber DA: Negative regulation of yap by lats1 underscores evolutionary conservation of the drosophila hippo pathway. Cancer Res 2008;68:2789-2794.

-33 Visser S, Yang X: Lats tumor suppressor: A new governor of cellular homeostasis. Cell Cycle 2010;9:38923903.

34 An Y, Kang Q, Zhao Y, Hu X, Li N: Lats2 modulates adipocyte proliferation and differentiation via hippo signaling. PloS one 2013;8:e72042.

-35 Kezele P, Nilsson E, Skinner MK: Cell-cell interactions in primordial follicle assembly and development. Front Biosci 2002;7:d1990-1996.

-36 Chen HJ, Wang CM, Wang TW, Liaw GJ, Hsu TH, Lin TH, Yu JY: The hippo pathway controls polar cell fate through notch signaling during drosophila oogenesis. Dev Biol 2011;357:370-379.

-37 Varelas X, Miller BW, Sopko R, Song S, Gregorieff A, Fellouse FA, Sakuma R, Pawson T, Hunziker W, McNeill H, Wrana JL, Attisano L: The hippo pathway regulates wnt/beta-catenin signaling. Dev cell 2010;18:579591.

-38 Yan Y, Denef N, Tang C, Schupbach T: Drosophila pI4kIIIalpha is required in follicle cells for oocyte polarization and hippo signaling. Development 2011;138:1697-1703. 


\section{Cellular Physiology Cell Physiol Biochem 2015;36:1712-1724 \begin{tabular}{l|l} 
Dor: 10.1159/000430144 & $\begin{array}{l}\text { 2015 S. Karger AG, Basel } \\
\text { www.karger.com/cpb }\end{array}$ \\
\hline
\end{tabular} \\ Li et al.: Ovarian Germline Stem Cells (OGSCs) Correlate with Hippo Signaling Pathway in Mice}

-39 Heallen T, Zhang M, Wang J, Bonilla-Claudio M, Klysik E, Johnson RL, Martin JF: Hippo pathway inhibits wnt signaling to restrain cardiomyocyte proliferation and heart size. Science 2011;332:458-461.

40 Kitadate Y, Kobayashi S: Notch and egfr signaling act antagonistically to regulate germ-line stem cell niche formation in drosophila male embryonic gonads. Proc Natl Acad Sci USA 2010;107:14241-14246.

41 Ward EJ, Shcherbata HR, Reynolds SH, Fischer KA, Hatfield SD, Ruohola-Baker H: Stem cells signal to the niche through the notch pathway in the drosophila ovary. Curr Biol 2006;16:2352-2358.

42 Xiang C, Li J, Hu L, Huang J, Luo T, Zhong Z, Zheng Y, Zheng L: Hippo signaling pathway reveals a spatiotemporal correlation with the size of primordial follicle pool in mice. Cell Physiol Biochem 2015;35:957968.

43 De Felici M: Germ stem cells in the mammalian adult ovary: Considerations by a fan of the primordial germ cells. Mol Hum Reprod 2010;16:632-636.

44 Ratajczak MZ, Machalinski B, Wojakowski W, Ratajczak J, Kucia M: A hypothesis for an embryonic origin of pluripotent oct-4(+) stem cells in adult bone marrow and other tissues. Leukemia 2007;21:860-867.

45 Zheng W, Zhang H, Gorre N, Risal S, Shen Y, Liu K: Two classes of ovarian primordial follicles exhibit distinct developmental dynamics and physiological functions. Hum Mol Genet 2014;23:920-928.

46 Bhartiya D, Singh J: FSH-FSHR3-stem cells in ovary surface epithelium: basis for adult ovarian biology, failure, aging, and cancer. Reproduction 2015;149:R35-48.

-47 Lian I, Kim J, Okazawa H, Zhao J, Zhao B, Yu J, Chinnaiyan A, Israel MA, Goldstein LS, Abujarour R, Ding S, Guan KL: The role of YAP transcription coactivator in regulating stem cell self-renewal and differentiation. Genes Dev 2010;24:1106-1118.

-48 Fu D, Lv X, Hua G, He C, Dong J, Lele SM, Li DW, Zhai Q, Davis JS, Wang C: YAP regulates cell proliferation, migration, and steroidogenesis in adult granulosa cell tumors. Endocr Relat Cancer 2014;21:297-310.

-49 Ataya KM, McKanna JA, Weintraub AM, Clark MR, LeMaire WJ:A luteinizing hormone-releasing hormone agonist for the prevention of chemotherapy-induced ovarian follicular loss in rats. Cancer Res 1985;45:3651-3656.

50 Meirow D, Nugent D: The effects of radiotherapy and chemotherapy on female reproduction. Hum Reprod Update 2001;7:535-543.

-51 Ruderman JV, Schmidt MR: RNA transcription and translation in sea urchin oocytes and eggs. Dev Biol 1981;81:220-228.

52 Sen GL, Wehrman TS, Blau HM: mRNA translation is not a prerequisite for small interfering rna-mediated mrna cleavage. Differentiation 2005;73:287-293.

53 Hatfield JM, Allen RG, Stack J, Ronnekleiv O: Post-translational processing of pro-opiomelanocortin (POMC)-derived peptides during fetal monkey pituitary development. II. beta-Lipotropin (beta-LPH)related peptides. Dev Biol 1988;126:164-172.

54 Tremblay AM, Camargo FD: Hippo signaling in mammalian stem cells. Semin Cell Dev Biol 2012;23:818826.

55 Ramos A, Camargo FD: The hippo signaling pathway and stem cell biology. Trends Cell Biol 2012;22:339346. 